\title{
Aerodynamic Modeling and Simulation of Flexible Wing Active Deformation based on Piezoelectric Material
}

\author{
Zhuo Han ${ }^{1,}$, , Zhengjie Wang, b) \\ ${ }^{1}$ School of Mechatronical Engineering, Beijing Institute of Technology, Beijing 100081, China. \\ a)849571743@qq.com \\ b)wangzhengjie@bit.edu.cn
}

\begin{abstract}
Flexible wing aircraft with the function of active deformation can improve the aerodynamic characteristics by the adaptive deformation of the airfoil, to maintain its own flight stability. In this paper, the active deformation of the flexible wing is realized by the piezoelectric material actuator, instead of the traditional servo control surface. This driving method can solve the issues about the continuous deformation of the airfoil and the timeliness of response well. The mechanical model of the piezoelectric composite flexible wing and the static aeroelasticity model of each wing panel are built in this paper. Based on the models, the coupling relationship among the piezoelectric force, the aerodynamic lift and the airfoil torsional deformation is obtained. According to the simulation results of the aerodynamic iteration optimization program AeroBeamSaw, the effect of the size and location of the active deformation on the aerodynamic lift is researched.
\end{abstract}

Keywords.piezoelectric material; flexible wing; active deformation; aerodynamic modeling and simulation

\section{INTRODUCTION}

Modern aircrafts require a good mobility within a wide range of velocity and altitude, but the aircrafts with traditional lifting device which improves the lift by changing the angle of the rudder are difficult to meet the requirements. The traditional lifting device will destroy the continuity of the airfoil, when the rudder deflects, so as to affect the aerodynamic characteristics of the wing ${ }^{[1]}$.

Over the years, aircraft designers have been expecting to improve the aerodynamic force by the deformation of the wing directly, instead of the rudder. Therefore, the concept of the active deformation wing is proposed: it means that the shape and curvature of the wing can be changed according to the needs of the task. The active deformation wing has a strong wing box, whose skin is made of fiber reinforced composite material. The drive components are embedded in the airfoil to drive the leading edge and trailing edge produce deformations. This type of the wing can motivate drive components, instead of the hinge structure, to make the wing twist or bend according to the flight condition and the bearing load of the structure. By this way, the airfoil and the attack angle can be changed, so as to obtain the best aerodynamic characteristics. Compared with the conventional control surface, the active deformation wing has a huge advantage and potential in reducing the weight of aircraft, simplifying the drive mechanism, reducing the critical load and increasing the lift ratio ${ }^{[2]}$.

Drive components are one of the key technologies of the active deformation wing. With the development of aerospace technology, intelligent material actuator has gradually replaced the traditional servo drive mode for some unique advantages. In this paper, the piezoelectric material is used as the drive component, which is bonded to the surface of the flexible wing. For the reverse piezoelectric effect, the piezoelectric actuator can make the airfoil produce torsional deformations to obtain the adaptive aerodynamic stability, so as to achieve the best aerodynamic characteristics ${ }^{[3]}$. 
The advantages of this drive mode are: (1) achieving the best torque layout and reducing the aerodynamic drag; (2) reducing the weight of the drive structure and simplifying the design; (3) suppressing the wing flutter and achieving the effect of gust alleviation; (4) improving the maneuverability and anti-disturbance of the aircraft ${ }^{[4]}$.

In this paper, the research object is the flexible wing with a wingspan of $1.7 \mathrm{~m}$. The content includes: deriving the relationship between the torque generated by the piezoelectric material and the torsion deformation of the airfoil; building the static aeroelasticity model of each wing panel; discussing the effect of the flexible wing active deformation on the aerodynamic characteristics of the wing according to the aerodynamic iteration simulation.

\section{AEROELASTICITY MODELING OF THE FLEXIBLE WING \\ Mechanical Model of the Piezoelectric Composite Flexible Wing}

The piezoelectric material is bonded to the upper surface of the flexible wing by the use of the paste method, and the piezoelectric composite flexible wing structure is formed, as shown in Figure 1. Due to the constraint of the wing structure, the output torque of the piezoelectric patches on the wing surface can generate the reaction force on the airfoil, resulting in driving force, to drive the airfoil deformed.

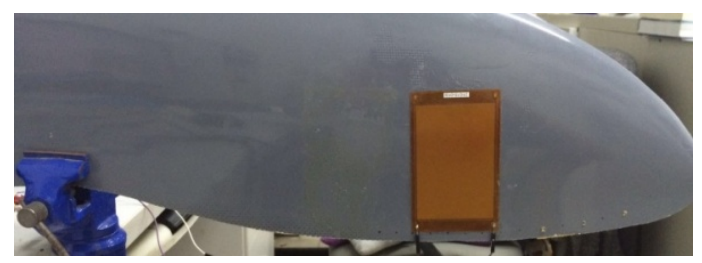

FIGURE 1. The piezoelectric composite flexible wing structure in this paper.

Due to the features of thin and curved for the flexible wing, the chord length of the airfoil section is much larger than the thickness, so the airfoil section can be equivalent to a narrow rectangular section. According to the theory of material mechanics ${ }^{[5]}$, the relationship between the output torque of the piezoelectric material and the torsional deformation of the airfoil can be obtained as:

$$
\delta=\frac{3 T y}{G_{s} b c^{3}}
$$

where $b$ and $c$ represent the chord length and thickness of the wing respectively; $T$ is the torque generated by the piezoelectric material; $G_{s}$ is the shear modulus of the material of the wing; $y$ is the distance between the deformation location and the wing root; and $\delta$ is the amount of the torsion deformation of the airfoil.

\section{The Effect of Active Deformation on Aerodynamic Lift}

The piezoelectric material actuator has replaced the traditional control surface to drive the airfoil deformed. In order to simplify the coupling between the piezoelectric force and the aerodynamic force, the piezoelectric actuator is equivalent to the control surface here. The binary wing model with a control surface is built, as shown in Figure 2, to research the effect of the active deformation on the aerodynamic lift.

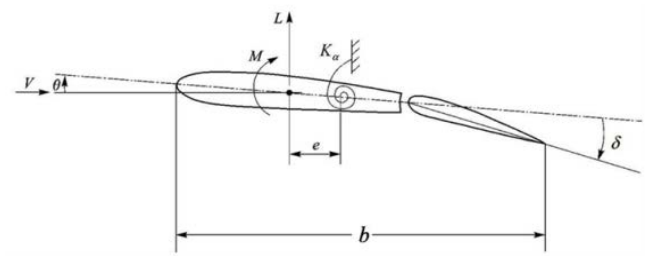

FIGURE 2. The binary wing model with a control surface. 
Let the deflection angle of the control surface be $\delta$. The aerodynamic lift acting on the aerodynamic center is:

$$
L=q S\left(\frac{\partial C_{L}}{\partial \alpha} \theta+\frac{\partial C_{L}}{\partial \delta} \delta\right)
$$

where $q$ is the dynamic pressure; $S$ is the reference area of the wing panel; $\frac{\partial C_{L}}{\partial \alpha}$ is the derivative of the lift coefficient to attack angle; $\frac{\partial C_{L}}{\partial \delta}$ represents the increment of the lift coefficient due to the unit deflection angle of the control surface; and $\theta$ is the flexible twist angle.

And then, according to the balance to the stiffness center, the linear relationship between the lift generated by the deflection of the control surface and the deflection angle can be obtained as:

$$
L=\frac{q S\left(\frac{\partial C_{L}}{\partial \delta}+\frac{q c S}{K_{\alpha}} \frac{\partial C_{L}}{\partial \alpha} \frac{\partial C_{M A C}}{\partial \delta}\right) \delta}{1-\frac{q e S}{K_{\alpha}} \frac{\partial C_{L}}{\partial \alpha}}
$$

where $e$ represents the distance between the stiffness center and the aerodynamic center, and $K_{\alpha}$ is the torsional stiffness coefficient.

It should be noted that the coefficient of $\delta$ contains two parts: the first is a pure aerodynamic part, which lead to the increase of lift with the increase of $\delta$, due to the changing of the airfoil camber; the second reflects the effect of the aeroelasticity. The negative pitching moment, which tends to reduce the attack angle, will be induced by the negative deflection of the control surface due to $\frac{\partial C_{M A C}}{\partial \delta}<0$, so as to reduce the lift. At a low airspeed, the lift increment caused by the deflection of the control surface has exceeded the reduction caused by the aeroelasticity effect, so the total lift will increase with the deflection angle of the control surface increasing. As the velocity increases, the aeroelasticity effect becomes stronger. When the velocity meet equation (4), the control surface is invalid, that is, no lift increment. The velocity at this moment is just the reversal velocity, which is also one of the constraints in the static aeroelasticity simulation.

$$
\frac{\partial L}{\partial \delta}=\frac{q S\left(\frac{\partial C_{L}}{\partial \delta}+\frac{q c S}{K_{\alpha}} \frac{\partial C_{L}}{\partial \alpha} \frac{\partial C_{M A C}}{\partial \delta}\right)}{1-\frac{q e S}{K_{\alpha}} \frac{\partial C_{L}}{\partial \alpha}}=0
$$

Therefore, in the design of flexible wing deformation, it is necessary to take full account of the problem of control reversal. The piezoelectric material actuator can be distributed at several locations along the chord and wingspan, to improve the reversal velocity as far as possible, by the cooperation deformation of several wing panels.

\section{Static Aeroelasticity Modeling}

The flexible wing is made of flexible materials, of which the aerodynamic load and mechanical behavior are affected by the aeroelasticity to a great extent. The aeroelasticity model involves the coupling relationship among aerodynamic force, inertial force, elastic force and control force, so it is very complicated in itself. At home and abroad, the existing modeling experience and very few software used to analyze the aeroelasticity with control method are only limited to control the rudder of the traditional wing. Therefore, when solving the case involving the active deformation of the flexible wing, only the static aeroelasticity model is the approach for the current theoretical level, that is, regardless of the coupling between the inertial force and the other three forces ${ }^{[6]}$.In this 
paper, the static aeroelasticity characteristics of the flexible wing is mainly researched, of which the elastic force is realized by the driving force of the piezoelectric material.

In order to facilitate the static aeroelasticity modeling of the flexible wing, it is divided into six wing panels along the wingspan, as shown in Figure 3.

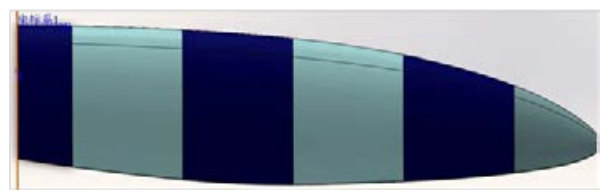

FIGURE 3. The distribution of the flexible wing along the spanwise.

According to the theory of static aeroelasticity, each panel can be equivalent to the cantilever structure. Regardless of the shear strain and the bending effect, the aeroelasticity equations of the flexible wing can be built $a^{[7]}$ :

$$
\begin{gathered}
E I_{i} \frac{\partial^{4} h}{\partial y^{4}}+C K_{i} \frac{\partial^{3} \phi}{\partial y^{3}}=\frac{\rho v^{2}}{2} S_{i} C_{L i}\left(\phi, \delta_{T E}, \delta_{L E}\right) \\
G J_{i} \frac{\partial^{2} \phi}{\partial y^{2}}+C K_{i} \frac{\partial^{3} h}{\partial y^{3}}=\frac{\rho v^{2}}{2} S_{i} C_{m i}\left(\phi, \delta_{T E}, \delta_{L E}\right)+\bar{q} S_{i} C_{l i} e_{i}
\end{gathered}
$$

where $E I_{i}, G J_{i}$ and $C K_{i}$ represent the bending stiffness coefficient, torsional stiffness coefficient and bendingtorsional coupling stiffness coefficient of NO. $i$ wing panel respectively; $h$ and $\phi$ represent the lateral displacement and torsion angle of each wing panel respectively; $C_{L i}$ and $C_{m i}$ are the lift coefficient and pitch moment coefficient of NO.i wing panel respectively; $\delta_{T E}$ and $\delta_{L E}$ are the deflection angles of the trailing edge and leading edge respectively; $e$ is the distance between the reference point of the pitch moment and the aeroelasticity center of each wing panel.

The static aeroelasticity equations of the whole wing can be written as the following matrix form ${ }^{[8]}$ :

$$
[K]\{d\}=\left\{A F\left(d, \delta_{T E}, \delta_{L E}\right)\right\}
$$

where $K$ is the stiffness coefficient matrix of the whole wing; $d$ is the deformation of the wing; $A F$ is the aerodynamic force acting on the airfoil, which is related to airfoil deformation and the deflection angles of the leading edge and trailing edge.

The aerodynamic calculation process AeroBeamSaw designed in this paper is an iterative optimization process. The whole iterative process of the static aeroelasticity is actually a process of coupling between the aerodynamic force and airfoil deformation. The leading edge and trailing edge of the wing generate elastic deformation $d$ drived by the piezoelectric material, which can be calculated by the equation (7) under this aerodynamic load. Then, the deformation $d$ generates additional aerodynamic force $A F$, which in turn makes the wing generate additional deformation, resulting in a new aerodynamic force. This process of mutual coupling iteration will gradually bring the flexible wing into balance. When the wing no longer deforms, that is, $d_{j+1}-d_{j}$ being less than the set value $\Delta d$, the aerodynamic force $A F$ is the needed one at this moment.

In this paper, the effect of the deformation size on the aerodynamic characteristics, such as lift, is obtained by iterative simulation, based on the aerodynamic iteration optimization program AeroBeamSaw. And airfoil deformation depends on the output torque of the piezoelectric material, so it also reflects the effect of the size and distribution location of the piezoelectric force on the aerodynamic force. 


\section{SIMULATION EXPERIMENT AND ANALYSIS}

The constraint condition of the simulation is the reversal velocity. Two groups of contrast experiments are designed to research the effect of the location distribution of the airfoil deformation along the chord and wingspan on the aerodynamic lift respectively.

\section{The Simulation of Chordwise Location Distribution}

In the first simulation experiment, the outermost wing panel with the largest deformation is selected. The trends of the aerodynamic lift varying with the deformation of the leading edge and railing edge at different velocities are obtained respectively, by the iterative simulation AeroBeamSaw, as shown in Figure 4 and 5.

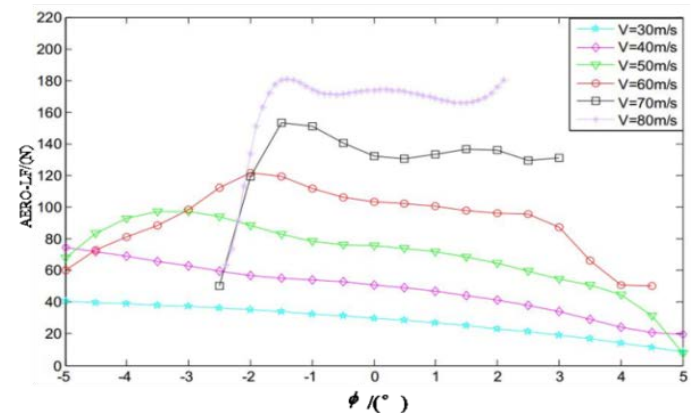

FIGURE 4. The curve of the aerodynamic lift varying with the deformation of the railing edge at different velocities.

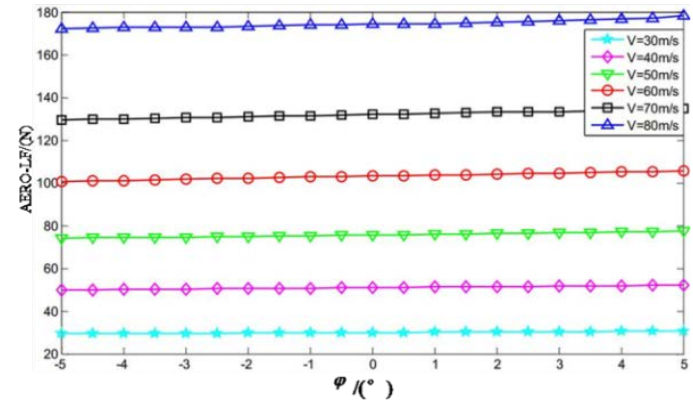

FIGURE 5. The curve of the aerodynamic lift varying with the deformation of the leading edge at different velocities.

It can be seen from the simulation results that the lift curve has an intersection when the deformation of the railing edge reaches $-2^{\circ}$, and the change of the deformation after the intersection is not obvious, which indicates that the reversal velocity is reached at this time. While, this phenomenon doesn't appear in the result of the leading edge, which indicates that the wing has a higher reversal velocity when the airfoil deformation is located at the leading edge. It also shows that the maximum deformation of the railing edge for the outermost wing panel is $\pm 2^{\circ}$.

\section{The Simulation of Spanwise Location Distribution}

In the other simulation experiment, the secondary wing panel is selected. Since the deformation of the outermost wing panel is the largest of all, of which the deformation range is $\pm 2^{\circ}$, the deformation of the secondary wing panel is set to $\pm 1^{\circ}$. The trends of the aerodynamic lift varying with the velocity at different deformations of the railing edge are obtained for the outermost and secondary wing panel respectively, by the iterative simulation AeroBeamSaw, as shown in Figure 6 and 7. 


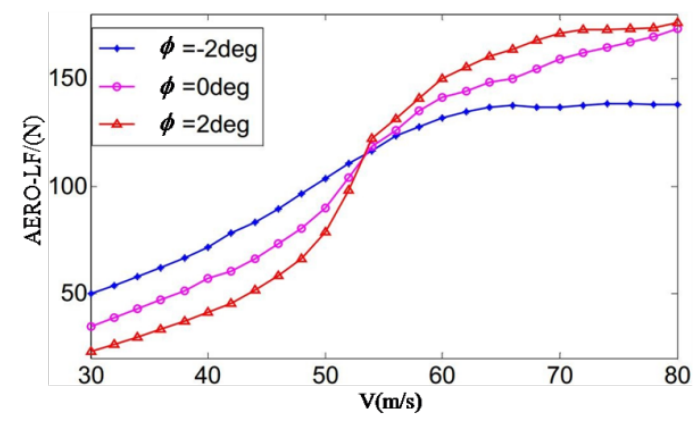

FIGURE 6. The curve of the aerodynamic lift varying with the velocity at different deformations for the outermost wing panel.

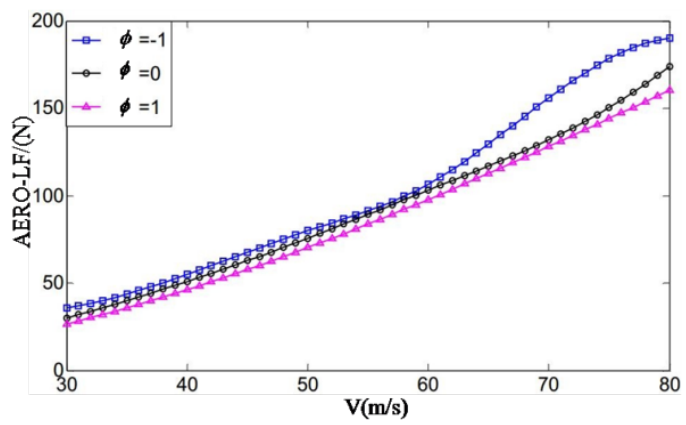

FIGURE 7. The curve of the aerodynamic lift varying with the velocity at different deformations for the secondary wing panel.

It can be seen from the simulation results that the lift curve of the outermost wing panel has an intersection at the velocity of $54 \mathrm{~m} / \mathrm{s}$, which is the reversal velocity. While, this phenomenon doesn't appear in the result of the secondary wing panel, which indicates that the wing has a higher reversal velocity, when the airfoil deformation is located at the secondary wing panel. Thus, it can be concluded that the closer the deformation location is to the wing root, the better the effect will have on improving the aerodynamic characteristics, and the higher the reversal velocity will be.

\section{CONCLUSION}

In this paper, the active deformation driven method, based on the piezoelectric material, can not only realize the function of the servo control surface, but also can solve the issues about continuous deformation of the airfoil and the timeliness of response. Based on the simulation results, it is concluded that it is more benefit to improve the aerodynamic characteristics, when the airfoil deformation is located at the leading edge of the wing and close to the wing root. Furthermore, it will have a better effect on improving the aerodynamic characteristics by the cooperation deformation of several piezoelectric material actuators, if they are installed on different locations of the wing. The further work of this paper is to build the experimental platform. The effect of the piezoelectric material actuator on driving the flexible wing active deformation to improve the aerodynamic characteristics under the real flight environment should be proved by the wind tunnel and flight testing.

\section{REFERENCES}

1. Boller C. Integrating shape memory alloys into composite materials: structural optimization and its implications on lightweight design[C]//Smart Materials, Structures, and Systems. International Society for Optics and Photonics, 2003: 1-9.

2. Tao Meizhen. Comprehensive Design of Modern Aircraft Structure [M]. Xi'an: Northwestern Polytechnical University Press, 2001

3. Stanford B, Sytsma M, Albertani R, et al. Static aeroelastic model validation of membrane micro air vehicle wings[J]. AIAA journal, 2007, 45(12): 2828-2837.

4. Yang Dazhi. Intelligent Material and Intelligent System [M]. Tianjin University Press, 2000. 
5. Han Bin, Liu Haiyan, Shui Xiaoping. Material Mechanics Course [M]. Electronic Industry Press, 2013.

6. Boothe K, Fitzpatrick K, Lind R. Controllers for disturbance rejection for a linear input-varying class of morphing aircraft[C]//46th AIAA/ASME/ASCE/AHS/ASC Structures, Structural Dynamics and Materials Conference. 2005: 2374.

7. Guo S. Aeroelastic optimization of an aerobatic aircraft wing structure[J]. Aerospace Science and Technology, 2007, 11(5): 396-404.

8. Liani E, GuoS, Allegri A. Potential-flow-based aerodynamic analysis and test of a flapping wing[C]//Proceedings of the 37th AIAA Fluid Dynamics Conference, Miami, Florida, AIAA-2007-4068. 2007. 